\title{
Within ratio responding during fixed ratio performance'
}

IVAN BAROFSKY AND DAVID HURWITZ, $U, S$ ARMY RESEARCH INSTITUTE OF ENVIRONMENTAL MEDICINE, Natick, Mass. 01760

Five rats were trained in steps from $F R 10$ to $F R 120$ or $F R$ 160. Response rate increased to a maximum between $F R 40$ or $F R$ 80 although the reinforcement frequency continually declined from $F R$ 10 to higher ratios. Measurement of interresponse times revealed that changes in within ratio responding were inversely correlated with changes in response rate.

The fixed ratio (FR) schedule of reinforcement (Ferster \& Skinner, 1957) characteristically generates a bimodal type of behavior, with periods of active responding (within ratio responding) and periods of complete absence of responding (postreinforcement pauses, PRPs). Most studies of FR performance, regardless of the experimental variable, account for changes in response rate primarily in terms of changes in postreinforcement pauses (e.g., Sidman \& Stebbins, 1954; Ferster \& Skinner, 1957; Premack, Schaeffer, \& Hundt, 1964; Felton \& Lyon, 1966). None of these studies include systematic measures of within ratio responding. Visual inspection of cumulative records reveal that within ratio responding remains relatively stable although "observable" within ratio pauses are noted (e.g., Felton \& Lyon, 1966).

The purpose of the present study was to determine if changes in within ratio responding were occurring during FR performance. Fixed ratio size was varied and interresponse times (IRTs) were used as measures of within ratio responding. Measurement of IRT was started at FR 40 and continued through higher ratios. The available literature indicated that response rate would decrease above FR 40 and that this reduction in response rate could be largely attributable to increases in PRPs (Ferster \& Skinner, 1957; Felton \& Lyon, 1966).

Subjects. Five male, experimentally naive, Sprague-Dawley rats served as experimental Ss. The animals were maintained at $80 \%$ of their ad lib body weight $(300-325 \mathrm{~g})$ throughout the experiment. Water was always available in the home cage.

Apparatus. The experimental chamber was a standard operant conditioning box with a lever, a light over the lever, and a food cup. Reinforcements (45 mg Noyes food pellets) were simultaneously accompanied by a brief onset of light $(0.5 \mathrm{sec})$ and a click of the food dispenser solenoid. The schedule was programmed on solid state modules and the data recorded on Gerbrand's cumulative records and strip recorders. The IRT frequency distribution in intervals of $190 \mathrm{msec}$ was recorded on 10 Sodeco ( 10 impulses per sec) counters. The second counter, for example, recorded all IRTs $\geqslant 191$ but $\leqslant 380 \mathrm{msec}$.

Procedure. The design of the experiment called for the initial establishment of stable behavior at FR 40 followed by the sampling of behavior at progressively higher ratios. The ratio size was increased by steps of 40 until response rate decreased and until observable within ratio pauses occurred, in the cumulative records of two consecutive ratio sizes.

All animals were initially trained by differential reinforcement of responses which successively approximated a lever press. Once the lever press occurred, the animals were allowed one or two sessions at FR 1; two sessions at FR 2, and four sessions at FR 5. A criterion of less than a 5\% variation in response rate between the first two and last two sessions of a successive fout-session period was used to progressively increase the fixed ratio size, in steps of 10 , from FR 10 to FR 40. The number of training sessions at FR 10 to FR 30 varied amongst animals as a function of the time required to meet the criterion of stable performance. The animals were trained at FR 40 for 90 days and at $F R 80$ for seven (animals $1 F, 2 \dot{F}$, and $3 F$ ) or eight (animals $4 F$ and $6 \mathrm{~F}$ ) days. Three animals $(1 \mathrm{~F}, 2 \mathrm{~F}$, and $3 \mathrm{~F})$ were trained at FR 120 for 18 days, while two animals ( $4 F$ and $6 F$ ) were trained at FR 120 for 10 days, and, since they maintained a high response rate with few observable within ratio pauses, they were also exposed to FR 160 for eight days.

A daily experimental session lasted $30 \mathrm{~min}$ (up to and including FR 10) or $1 \mathrm{~h}$ (beyond FR 10). The animals were trained seven days a week until they were exposed to FR 80 at which time they were trained five days a week.
Results. Analysis of the response rate, IRTs, and frequency of reinforcement were based on the mean of four days data at the end of each ratio size exposure for each animal. PRPs were not collected in the present experiment, but inspection of cumulative records suggested that longer PRPs occurred as FR size increased.

The mean response rate (responses/sec) for each animal is plotted as a function of fixed ratio size in Fig. 1. Response rate increased to a maximum at FR 40 or FR 80 with relatively small declines at higher ratio sizes. The mean reinforcements per $1 \mathrm{~h}$ session (Fig. 2) declined in a negatively accelerated fashion with increases in ratio size.

The IRT distributions were calculated on the basis of the frequency of entries in the first nine $(190 \mathrm{msec})$ intervals. This procedure excluded all IRTs greater than $1.71 \mathrm{sec}$ but included any PRP less than $1.71 \mathrm{sec}$ long. The first three IRT intervals (i.e., IRTs $\leqslant 570 \mathrm{msec}$ ) contained $87-97 \%$ of all IRTs. The second interval (191-380 msec) was the modal interval at all ratio sizes for four of the animals. The first interval $(0-190 \mathrm{msec})$ was the modal interval for a fifth animal $(6 \mathrm{~F})$ at all ratios except FR 160, where the mode shifted to the second interval. Although the modal interval remained constant as ratio size increased, changes occurred in the absolute and relative frequencies of IRTs in adjacent intervals.

To evaluate whether these changes in the IRT distributions were associated with changes in response rate, the mean IRT estimated from all nine IRT intervals or the first three IRT intervals, and the median estimated from all nine IRT intervals were calculated at any one ratio size and correlated with the corresponding response rates for all the animals. Statistically significant Pearson product moment correlation coefficients were found for each comparison with $r=-.85$ for the mean of nine intervals, $r=-.81$ for the mean of three intervals, and $r=-.80$ for the median ( $d f=16, p<.01$, for each correlation). The demonstration that the mean IRT for the first three intervals correlates equally as highly as the correlation based on all nine intervals suggests that most of the changes in response rate are due to changes in the frequency of IRTs $\leqslant 590$ msec. It can be concluded from this analysis that changes in within ratio responding, as measured by IRTs, are highly correlated with response rate changes at and above FR 40.

Discussion. In the present experiment, response rate increased to a maximum at FR 40 or FR 80 (depending on the animal) and then declined at higher ratios. These results confirm and extend the observations of Boren (1953) with rats, and replicate the studies of Ferster \& Skinner (1957) and

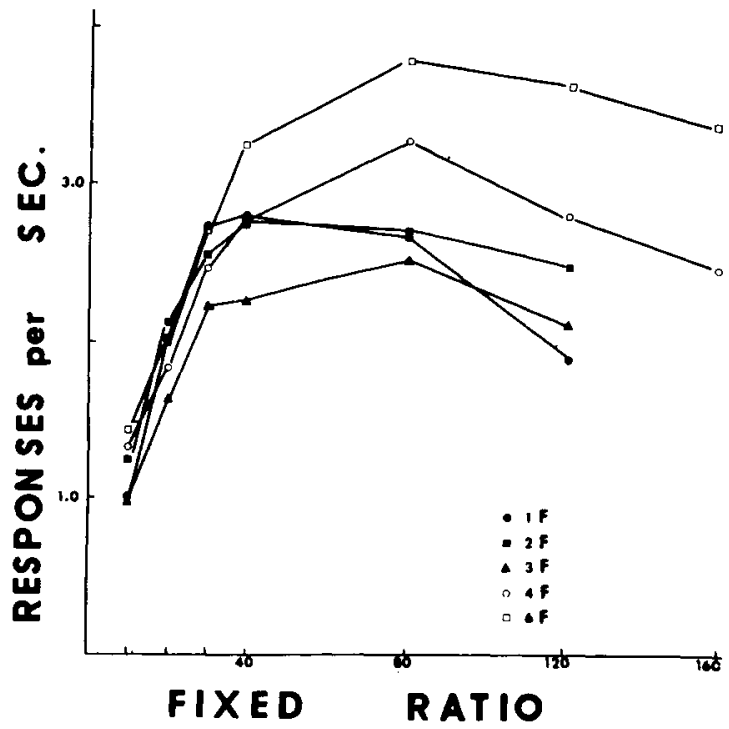

Fig. 1. Responses per second are plotted as a function of fixed ratio for each animal. Each point represents a mean of four days. 


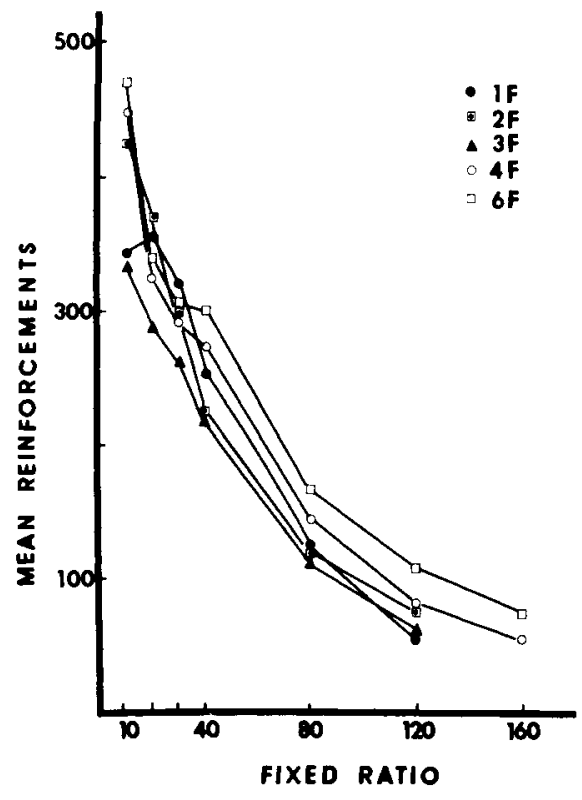

Fig. 2. The mean number of reinforcements per session are plotted as a function of fixed ratio sized and individual animal.
Felton \& Lyon (1966) with pigeons. Comparison of Figs. 1 and 2 demonstrates that the response rate was, in general, inversely correlated with the frequency of reinforcement. This contrasts with the direct relationship found between response rate and reinforcement frequency in fixed or variable interval schedules of reinforcement (Morse, 1966).

The correlations found between the mean or median IRTs and response rate demonstrates that, under the present experimental condition, changes in within ratio responding account for $64-72 \%$ (depending on the measure) of the variation in response rate. Error and PRP variance would then account for the remaining variance. Thus, it appears that changes in both PRP and within ratio responding should be considered in evaluating how changes in overall response rate occur during $F R$ performance.

\section{REFERENCES}

BOREN, J. J. Response rate and resistance to extinction as function of the fixed ratio. 1953, Doctoral Dissertation, Columbia University, New York, N. Y.

FELTON, M., \& LYON, D. O. The postreinforcement pause. J. exp. Anal. Behav., 1966, 9, 131-134.

FERSTER, C. B., \& SKINNER, B. F. Schedules of reinforcement. New York: Appleton-Century-Crofts, 1957.

MORSE, W. H. Intermittent reinforcement. In W. K. Honig (Ed.), Operant behavior: Areas of research and application. New York: AppletonCentury-Crofts, 1966.

PREMACK, D., SCHAEFFER, R W., \& HUNDT, A. Reinforcement of drinking by running: Effect of fixed ratio and reinforcement time. J. exp. Anal. Behav., 1964, 7, 91-96.

SIDMAN, M., \& STEBBINS, W. C. Satiation effects under fixed-ratio schedules of reinforcement. J. comp. physiol. Psychol., 1954, 47, 114-116. NOTE

1. A portion of this research was presented at the April 1967 meetings of the Eastern Psychological Association. 